\title{
Neural basis for inferring false beliefs and social emotions in others among individuals with schizophrenia and those at ultra-high risk for psychosis
}

(A revised manuscript to be submitted as PSYN_2016_45R2 in Psychiatry Research: Neuroimaging)

Yosuke Takano $^{\mathrm{a}}$, Yuta Aoki ${ }^{\mathrm{a}, \mathrm{b}}$, Noriaki Yahata ${ }^{\mathrm{a}}$, Yuki Kawakubo ${ }^{\mathrm{c}}$, Hideyuki Inoue ${ }^{\mathrm{a}}$, Norichika Iwashiro $^{a}$, Tatsunobu Natsubori ${ }^{\mathrm{a}}$, Shinsuke Koike ${ }^{\mathrm{a}}$, Wataru Gonoi ${ }^{\mathrm{d}}$, Hiroki Sasaki ${ }^{\mathrm{d}}$, Hidemasa Takao $^{\mathrm{d}}$, Kiyoto Kasai ${ }^{\mathrm{a}}$ and Hidenori Yamasue, ${ }^{\mathrm{a}, \mathrm{e}^{*}}$

${ }^{a}$ Department of Neuropsychiatry, Graduate School of Medicine, The University of Tokyo, 7-3-1 Hongo, Bunkyo-ku, Tokyo 113-8655, Japan

${ }^{\mathrm{b}}$ The Child Study Center at NYU Langone Medical Center, One Park Avenue, New York, NY 10016, USA

${ }^{\mathrm{c}}$ Department of Child Neuropsychiatry, Graduate School of Medicine, The University of Tokyo, 7-3-1 Hongo, Bunkyo-ku, Tokyo 113-8655, Japan

${ }^{\mathrm{d}}$ Department of Radiology, Graduate School of Medicine, The University of Tokyo, 7-3-1 Hongo, Bunkyo-ku, Tokyo 113-8655, Japan

e Department of Psychiatry, Hamamatsu University School of Medicine, 1-20-1 Handayama, Higashiku, Hamamatsu City 431-3192, Japan

*Corresponding author: Department of Psychiatry, Hamamatsu University School of Medicine, 1-20-1 Handayama, Higashiku, Hamamatsu City 431-3192, Japan

Tel.: +81-53-435-2295

Fax: +81-53-435-3621

E-mail: yamasue@hama-med.ac.jp

Abstract: 200 words

Text body: 4585 words

Acknowledgements: 48 words

Figure legends: 80 words; 1 figure; 2 tables; 4 supplementary materials 


\begin{abstract}
Inferring beliefs and social emotions of others has different neural substrates and possibly different roles in the pathophysiology of different clinical phases of schizophrenia. The current study investigated the neural basis for inferring others' beliefs and social emotions, as individual concepts, in 17 subjects at ultra-high risk for psychosis (UHR), 16 patients with schizophrenia and 20 healthy controls. Brain activity significantly differed from normal in both the left superior temporal sulcus (STS) and the inferior frontal gyrus (IFG) in the schizophrenia group while inferring others' beliefs, whereas those of UHR group were in the middle of those in the schizophrenia and healthy-control groups. Brain activity during inferring others' social emotions significantly differed in both the left STS and right IFG among individuals at UHR; however, there was no significant difference in the schizophrenia group. In contrast, brain activity differed in the left IFG of those in both the schizophrenia and UHR groups while inferring social emotion. Regarding the difference in direction of the abnormality, both the UHR and schizophrenia groups were characterized by hyper-STS and hypo-IFG activations when inferring others' beliefs and emotions. These findings might reflect different aspects of the same pathophysiological process at different clinical phases of psychosis.
\end{abstract}

Key Words: Empathy, Inferior frontal gyrus, Mentalizing, Social cognition, Superior temporal sulcus, Theory of Mind 


\section{Introduction}

Social cognitive impairments among people with schizophrenia are consistently observed across distinct phases of illness (Green et al., 2012) and are considered an important determinant of prognosis and functional outcome (Fett et al., 2011; Horan et al., 2012; Schmidt et al., 2011; Smith et al., 2015). Therefore, this phenotype has received attention as a promising treatment target and surrogate endpoint of clinical trials (Green et al., 2013; Green and Penn, 2013; Kern et al., 2013; Olbert et al., 2013). Among deficits across social cognitive domains (Savla et al., 2013), deficits in inferring others' mental statuses, known as theory of mind (ToM) have been emphasized in light of their relationship to some specific schizophrenia symptoms. These symptoms include delusions of persecution and of reference, third-person auditory hallucinations, thought disorder and negative symptoms (Frith, 1992; Ventura et al., 2015; 2013).

Inferring others' mental statuses can be separated into two distinct subcomponents: inferring others' beliefs and emotions (Shamay-Tsoory, 2011; Vollm et al., 2006; Zaki and Ochsner, 2012). Previous studies have suggested that there are distinct neural bases underlying the deficits of inferring beliefs and those of inferring emotions in patients with schizophrenia (Derntl et al., 2012; Harvey et al., 2013; Modinos et al., 2010). A limited number of studies have investigated the neural bases of these deficits using single functional magnetic resonance imaging (fMRI) tasks in the same participants (Benedetti et al., 2009; Lee et al., 2010). Statistical methods and the subsequent results vary between studies. These studies suggest altered function in right superior temporal regions of participants with schizophrenia during the inference of others' belief and/or emotions (Benedetti et al., 2009; Lee et al., 2010). However, these studies used different stimuli in their psychological tasks between inferring beliefs and emotions to examine their neural bases. Thus, the contrasts in these studies may not precisely differentiate the neural basis for the inference of others' emotions or beliefs. It is important to be able to distinguish whether 
these two deficits have independent neural bases and different relationships to functional outcomes, to provide evidence for their potential use as biomarkers in future therapeutic studies. In our recent study, we demonstrated the presence of differences in the neural correlates for each subcomponent together with their potential for use as therapeutic targets (Aoki et al., 2014).

Individuals who eventually develop psychotic disorders, such as schizophrenia, often suffer initially from several social and/or cognitive deficits. Individuals presenting with these deficits but not meeting full criteria for schizophrenia are thought to be at ultra high risk (UHR) for schizophrenia. Some of these UHR individuals will go on to develop schizophrenia. Therefore, investigating neural differences between individuals at UHR and those with schizophrenia may provide insight into the neural basis of the development and progression of the disorder (Pantelis et al., 2009). In fact, we have previously reported implicit differences in the functional and neuroanatomical correlates of the clinical stages of psychosis in brain regions such as the inferior frontal, superior temporal and medial prefrontal cortices (Iwashiro et al., 2012; Natsubori et al., 2014a; Natsubori et al., 2014b; Suga et al., 2010; Yamasue et al., 2004).

UHR individuals have demonstrable deficits in ToM, and neuroimaging studies have reported abnormalities in corresponding brain regions (Lee et al., 2013; Schurz et al., 2014). However, no study has yet examined the neural correlates that distinguish the processes of inferring others' beliefs from that of inferring others' emotions in people with schizophrenia or UHR. Given that there are abnormalities in ToM brain regions that are dependent on clinical stage in patients with schizophrenia and given that UHR individuals show cognitive ToM deficits, we hypothesized the presence of clinical stage-dependent functional abnormalities in patients with schizophrenia or UHR.

The current study employed an event-related fMRI task design to investigate brain activity related to the processes of inferring others' beliefs and emotions in response to the same 
stimuli in patients with schizophrenia and in those at UHR. We employed a modified psychological paradigm (based on the Sally-Ann task, a first-order false-belief task (Aoki et al., 2014)), which was recently developed by our research group, to identify different neural correlates of inferring others' beliefs and emotions. This task presents a situation in which one person has cheated the other. The one who cheated gloats after successfully deceiving the other, who is frustrated. The emotions in this task are therefore social emotions rather than basic emotions. Based on the previous literature outlined above, we predicted that brain regions such as the inferior frontal, superior temporal and medial prefrontal cortices would be associated with the inference of others' mental states. We also hypothesized that these neural correlates would be related to schizophrenia symptoms.

\section{Methods}

\subsection{Participants}

\section{Insert Table 1 about here}

We enrolled 53 right-handed Japanese participants in this study. Handedness was evaluated using the Edinburgh Handedness Inventory (Oldfield, 1971). The recruitment site, inclusion/exclusion criteria for each diagnosis, and the clinical and demographic assessment methods were the same as those used in our previous studies (Iwashiro et al., 2012; Koike et al., 2013; Natsubori et al., 2014a; Natsubori et al., 2014b). Briefly, the inclusion criteria for UHR were aged 15-30 years and a diagnosis of UHR for psychosis based on the Structured Interview for Prodromal Symptoms (SIPS) (Kobayashi et al., 2007; Miller et al., 1999). Schizophrenia was diagnosed using the Structured Clinical Interview for the Diagnostic (SCID-1) and Statistical Manual of Mental Disorders, Fourth Edition, Axis I Disorders, Clinical Version (First MB et al., 1997). Psychiatric symptoms were evaluated using the Positive and Negative Syndrome Scale (PANSS) (Kay et al., 
1987) within 14 days (7dayts before and 7days after MRI scan). The clinical group comprised 33 participants, of which 17 individuals were at UHR and 16 had schizophrenia. Among the UHR participants, 11 had attenuated positive symptom syndrome (APSS), one had brief intermittent psychotic syndrome (BIPS), three had genetic risk and deterioration syndrome (GRDS), one had APSS and GRDS, and one had BIPS and APSS. These diagnoses were based on the SIPS criteria. Another participant had comorbid schizophreniform disorder. No UHR participants had any other comorbid psychiatric diagnoses. Antipsychotics were prescribed for nine participants at UHR and for all participants with schizophrenia. Twenty healthy controls (HCs) were recruited from internet referral, message board in several universities and voluntary recruitment in the University of Tokyo Hospital. The absence of any psychiatric condition in control participants was confirmed using the SCID-1 non-patient edition and PRIME-Screen. There were no significant differences in age, gender, parental socioeconomic-status (Hollingshead, 1965) or intelligence quotients (Matsuoka et al., 2006; Uetsuki et al., 2006) between the three groups (Table 1). All clinical evaluations were performed by a trained psychiatrist (T.N., N.I., or Y.T.).

The exclusion criteria for all groups were a current or past neurological comorbidity, traumatic brain injury with any known cognitive consequences or loss of consciousness for more than 5 min, a history of electroconvulsive therapy, and substance abuse or addiction. An additional exclusion criterion for the control group was any history of neuropsychiatric disorder in the participants or any history of axis I disorders in first-degree relatives.

The ethical committee of the University of Tokyo Hospital approved the current study (397/1350/2226). After a complete explanation, written informed consent was obtained from all participants and from the parent if the participant was under 20-years-old.

\subsection{MRI scanning}


A 3T MRI scanner (GE Signa HDxt, Milwaukee, WI, USA) in the University of Tokyo Hospital was used. The anatomical scanning sequence was a 3D Fourier-transform spoiled-gradientrecalled acquisition $($ repetition time $=6.8 \mathrm{~s}$, slice thickness $=1 \mathrm{~mm}$, in-plane resolution $=1 \times 1 \mathrm{~mm}$ ). One of three trained neuroradiologists (H.T., H.S., or W.G.) evaluated the MRI scans to confirm the absence of gross abnormality. Gradient-echo, echo-planar sequences were used for functional imaging (repetition time $=3 \mathrm{~s}$, echo time $=35 \mathrm{~ms}$, flip angle $=80^{\circ}, 4 \times 4 \times 4 \mathrm{~mm}^{3}, 42$ slices, ventralto-dorsal interleaved acquisition). One run lasted $7 \mathrm{mins} 30 \mathrm{sec}$. One examination consisted of two such runs. The first ten functional volumes were excluded from analysis for the equilibrium of longitudinal magnetization.

\subsection{Experimental procedure and paradigm during fMRI}

In the present study, we used the same fMRI procedure and paradigm as in our previously published study (Aoki et al., 2014). Briefly, during an event-related fMRI scan, participants underwent two runs consecutively. During one run, ten different stories were presented (Supplementary Figure 1). Each story was presented three times consecutively, and participants were required to answer one yes/no question at the end of each presentation. Questions to which the answer was 'yes' in the first run were inverted such that in the second run the correct answer to these questions was 'no'. Questions that were answered 'no' in the first run were also inverted in the second run. The order of questions was determined in a pseudorandom fashion. Each story consisted of four comic frames based on stories about 'Sally' and 'Anne' (Baron-Cohen et al., 1985; Senju et al., 2009). Before the scan, all the participants practiced the psychological task in the scanner. During the practice, participants were shown cartoon stories and required to answer questions that had been modified from those used in the 'control' condition of the main psychological task. Thus, they were trained to understand the cartoon stories to complete the task 
appropriately without experiencing the questions of 'belief' and 'social emotion' conditions in the task.

As shown in Supplementary Figure 1, each trial consisted of (1) a 3.5-s story with four frames; (2) a 2-s frame with a written question; (3) a 2.5- to 3.5-s fixation cross; (4) a 3-s frame with a comic vignette was shown. In the vignette, an arrow pointed to the person of interest and a star indicated the location of interest to help the participant answer the question. (5) a 3-s fixation cross. Participants were required to push a button to answer yes or no during the last frames with the comic and fixation cross. The reaction time (RT) and correct rate (CR) were measured during this task. The four frames show a story with two characters with emotionally neutral faces. In the stories, one protagonist (Sally) places the object (e.g. ball) in a blind spot (e.g. glass) (Frame 1). While Sally is blindfolded (Frame 2), the other character (Anne) moves the object into another blind spot (e.g. under the desk) (Frame 3) before Sally takes the blindfold off Anne (Frame 4).

Ten stories were each presented three times, along with one of three different questions (to assess control, belief or social emotion). These 30 stimuli (10 stories $\mathrm{x}$ three questions) were presented in a psuedo-random order: (1) 'Is the ball actually in this place?' (a blind spot is indicated), as a control; (2) 'Does she look for her ball in this place?' (a blind spot is indicated), to stimulate belief inference and (3) 'Does she feel playful after seeing the glass opened?' to stimulate social emotion inference. For the first question, the participants were required to answer the actual location of the ball. Thus, this condition was utilized as a control. For the second question, participants were required to understand that Sally's action is based on what she believes to be true rather than what actually is. To answer this question, it is necessary both to understand the first-order false belief and to figure out the actual location of the object. Thus, we considered this to reflect belief inference. For the third question, participants were required to comprehend Anne's emotional status after noticing that Sally had performed a prank. To imagine 
Anne's emotional status rationally, the participants needed to understand that Anne deems that Sally falsely believes that the ball is in the cup, when it is in fact under the desk. Hence, to infer Anne's social emotion correctly, the following three cognitive components are necessary: understanding the second-order false belief (Shamay-Tsoory et al., 2009), understanding the firstorder false belief and actual location of the object and the ability to infer others' social emotions. We therefore considered this to reflect social emotion inference.

Given that belief inference requires understanding of the first-order false belief and of the actual location, which is required to answer the control question, the difference between the questions governing belief inference and control represents the ability to infer others' false beliefs. The brain activity was termed the 'belief-minus-control activity'. In contrast, the difference between the social emotion inference and belief inference conditions represents the ability to identify the first- and second-order false beliefs in addition to inferring others' social emotions, and the brain activity was termed 'social_emotion-minus-belief activity'.

\subsection{Behavioral analysis}

For group comparisons of behavioral data in the task, we performed repeated-measures analysis of variance (ANOVA) using either RT or CR as the dependent variable, the groups (HC, UHR, or schizophrenia) as the between-subject factors and the conditions (control, belief or social emotion) as a within-subject factor. The statistical significance level was set at $P<0.05$. All statistical analyses of behavioral data were conducted using SPSS statistics, Version 17.0, (SPSS Institute Inc., Chicago, IL, USA).

\subsection{Analysis by fMRI}

The fMRI data were analyzed using SPM8 (The Wellcome Department of Imaging Neuroscience, 
London, UK) running MATLAB 2009a (MathWorks Inc., Natick, MA, USA). Functional images were realigned, slice-timing corrected, normalized to the default echo-planar imaging template, smoothed (full width half maximum= $8 \mathrm{~mm}$, Gaussian filtered) and high-pass temporal filtered with a cut-off of $128 \mathrm{~s}$ to remove low-frequency drift from the data.

At the single-subject level, we used a general linear model with three regressors (control, belief or social emotion). Each event-related regressor had an onset at the time of stimulus (presentation of question) and a duration that corresponded to each RT. The six motion parameters resulting from realignment $(\mathrm{x} / \mathrm{y} / \mathrm{z} / \mathrm{pitch} / \mathrm{yaw} /$ roll $)$ were also included as regressors to account for residual effects of head motion. We estimated belief-minus-control activity and social_emotion-minus-belief activity (contrast images of interest) for each participant. We analyzed brain activity only for correctly answered questions.

Small volume correction was applied to assess the differences in hemodynamic response magnitude when inferring others' beliefs and social emotions, using the automated anatomical labeling tool in WFU Pickatlas. The mask included the bilateral "Frontal_Sup_Medial," “Cingulum_Ant," "Parietal_Inf," "SupraMarginal," "Angular," “Temporal_Pole_Sup," “Temporal_Pole_Mid," “Temporal_Sup," "Heschl," Frontal_Inf_Oper," "Frontal_Inf_Tri," "Insula," "Amygdala" and "Precuneus." These regions were chosen based on related previous studies reporting neural correlates of these cognitive components (Benedetti et al., 2009; Derntl et al., 2012; Harvey et al., 2013; Lee et al., 2010; Smith et al., 2015).

We performed second level analyses, using a full factorial model, to examine the effects of the following: group (HC, UHR or Schizophrenia), contrast (belief-minus-control or social_emotion-minus-belief) and group-by-contrast interaction. The statistical threshold was set at $P<0.001$, uncorrected for multiple comparisons with a minimum cluster extent of ten voxels, based on recent fMRI studies in patients with schizophrenia or those at clinical high risk with 
similar sample sizes (Choi et al., 2012; Das et al., 2012; de Achaval et al., 2012; Holt et al., 2011). The analysis identified brain regions where individuals with UHR, schizophrenia and HC had shown different activities between belief-minus-control and social_emotion-minus-belief. The blood-oxygen-level-dependent (BOLD) signal of the peak coordinate was extracted. The mean of BOLD signal was defined as the "signal intensity" for the contrast of interest. Signal intensity was then used to assess the group differences and correlations with clinical indices. As post-hoc analyses, we performed two-sample $t$-tests between groups. The significance level was set at $P<0.05$.

\subsection{Correlation between signal intensity and clinical indices}

We explored the potential association between symptom severity and the difference in BOLD signals in the peak coordinates of significant interaction or the main group effect, using Pearson's correlation coefficients in the UHR and schizophrenia groups. Symptom severity included PANSS scores (positive, negative and general psychopathology) and delusional behavior scores (PANSS P1 [delusion]+P5 [grandiosity]+P6 [suspiciousness]+G9 [unusual thought content] (Suga et al., 2010; Sumich et al., 2005; Yamasaki et al., 2007)). Correlations between the BOLD signals and antipsychotic doses were also examined to assess the potential confounding effects of antipsychotics. Considering the exploratory nature of the analyses, the statistical threshold was set at $P<0.05$.

\section{Results}

\section{Insert Table $2 \&$ Figure 1 about here}

\subsection{Behavioral data (Supplementary Table 1)}

The ANOVA showed no significant interaction between group and condition on the CR 
$(F[4,100]=0.9, P=0.44)$ and no significant main effect by group $(F[2,50]=2.3, P=0.11)$; in contrast, there was a significant main effect by condition $(F[2,100]=16.0, P<0.001)$. With regard the RT, there was no significant interaction between group and condition $(F[4,100]=0.6, P=0.60)$ and no significant main effect by group $(F[2,50]=1.8, P=0.17)$, but there was a significant main effect by condition $(F[2,100]=42.3, P<0.001)$. These results indicate that there was no significant difference in behavioral performance between diagnostic groups.

\subsection{Analysis by fMRI}

A significant main effect by group was found in the bilateral inferior frontal gyri (IFG) (Right: [46, 28, 22, Montreal Neurological Institute coordinates], $k=34, Z=3.6$; Left: $[-48,20,18]$, $\mathrm{k}=130, Z=3.8)$ and left superior temporal sulcus (STS) $([-50,-28,2], \mathrm{k}=54, Z=4.1)$ (Figure 1, Table 2).

The post-hoc analyses revealed that the UHR group showed significantly less social_emotion-minus-belief activity in the right IFG compared with the $\mathrm{HC}(t(35)=3.8, P=0.003)$ and schizophrenia $(t(31)=2.7, P=0.011)$ groups. However, the UHR $(t(35)=3.2, P=0.003)$ and schizophrenia $(t(34)=3.0, P=0.005)$ groups showed significantly less social_emotion-minus-belief activity in the left IFG than that in the HC group. The UHR group also showed significantly more social_emotion-minus-belief activity in the left STS than that in the HC group $(t(35)=2.6$, $P=0.014)$. Meanwhile the schizophrenia group did not show a significant difference in social_emotion-minus-belief activity in the STS compared with either the HC or UHR group. Regarding belief-minus-control contrast, the schizophrenia group showed significantly less activity in the left IFG than that in the HC group $(t(34)=2.1, P=0.043)$. However, the schizophrenia group showed significantly more belief-minus-control activity in the left STS than that in the $\operatorname{HC}(t(34)=3.8, P=0.001)$ and UHR $(t(31)=2.1, P=0.040)$ groups. 
The main effects of contrast were found in several clusters in the frontal, temporal and parietal cortices (Supplementary Table 2). No significant interaction was found between group and contrast. None of the significant effects survived correction for multiple comparisons.

\subsection{Correlation between signal intensity and clinical indices}

Pearson's correlation analysis showed that the elevated belief-minus-control activity in the left STS of individuals at UHR was significantly correlated with the higher PANSS general psychopathology $(r=0.66, P=0.004,95 \%$ confidence intervals $(\mathrm{CI}) 0.26$ to 0.86 ), positive symptoms ( $r=0.53, P=0.029,95 \% \mathrm{CI} 0.07$ to 0.81$)$ and delusional behavior $(r=0.60, P=0.012$, $95 \% \mathrm{CI} 0.16$ to 0.84$)$ scores. In contrast, there were no significant correlations between clinical symptoms and social_emotion-minus-belief activity in any brain region, in activity of any IFG contrast in the UHR group, in the activity of any contrast in any region in the schizophrenia group, or between negative symptoms and the activity of any contrast in any region in both clinical groups. However, Fisher's r-to-z transformation revealed that the correlation coefficients between brain activity and symptom severity were not significantly different $(P>0.26)$. The activity also exhibited no significant correlation with antipsychotic dose in either clinical group.

\section{Discussion}

In this study, we showed that both the UHR and schizophrenia groups were characterized by hyper-STS activity and hypo-IFG activity when engaged in inferring others' beliefs and/or social emotions. The schizophrenia group showed higher activity in the left STS than that of either the HC or UHR group when engaged in inferring others' beliefs. On the other hand, the UHR group showed higher activity in this brain region when inferring others' social emotions. In contrast, in the schizophrenia group, significantly lower activity was observed in the left IFG when patients 
were engaged in inferring others' beliefs. With regard to IFG activity while inferring others' social emotions, both the UHR and schizophrenia groups showed lower activity in the left IFG, and the UHR group showed lower activity in the right IFG than that of the HC and schizophrenia groups. Of note, although no correlations were found for any of the clinical symptoms with the IFG activities, the high left STS activity correlated with the severer psychotic symptoms among individuals at UHR.

We identified hyperactivation of the left STS when patients in the UHR and schizophrenia groups were engaged in inferring others' beliefs; also, when inferring others' social emotions, in this brain region, the UHR group showed higher activity from that in the HC group. These results are consistent with those of previous fMRI studies in patients with schizophrenia, implementing psychological tasks that required others' mental states to be inferred (Benedetti et al., 2009; Das et al., 2012). Among the few fMRI studies in individuals at UHR, our results are most consistent with those of Brune et al., who found higher activity in the left STS in a UHR group than that in $\mathrm{HC}$ and schizophrenia groups during an intention reading task (Brune et al., 2011).

We investigated the potential relationship between neural ToM disturbances and severity of delusions, assessed using the PANSS. This is because we assume that rational interpretations of situations require ToM. Elucidating disturbances at the neural level may provide insight into the pathophysiology underlying the development of schizophrenia. It is also noteworthy that the clinical correlates of the current neuroanatomical findings are confined to the STS. In the left STS of participants in the UHR group, the presence of higher activity while inferring others' beliefs correlated with higher PANSS scores for general psychopathology, positive symptoms and delusional behavior domains. Although there were no significant differences in behavior when inferring others' beliefs between the HC and UHR groups, the high activation may represent a 
transient neurological disturbance that leads to the development of schizophrenia. In fact, a number of previous studies have reported that the superior temporal cortices were associated with time-course changes during illness progression in schizophrenia (Kasai et al., 2003; Takahashi et al., 2009), lending support to this interpretation. One explanation for the correlation between hyper-STS activity and psychotic symptoms in the UHR group is that over-mentalizing contributes to shape the assessment of others' beliefs, and therefore, to the potential for misreading (Frith, 2004). In contrast, chronic medication and stable symptom conditions in the schizophrenia group might have blurred the relationship between clinical symptoms and brain activity in the current study. It should be noted that some previous studies have suggested that ToM is associated with the negative rather than positive symptoms of schizophrenia (Ventura et al., 2015; 2013). Further, it has been reported that brain activity in regions associated with ToM, especially the medial prefrontal cortex (mPFC), is related to social anhedonia (Dodell-Feder et al., 2014). The lack of significant changes in the mPFC in the present study suggests that this region may support social motivation linked with social anhedonia, but not with the more general negative symptoms measured by PANSS. In addition, other neural correlates may also regulate the support of social motivation by the mPFC. However, these possibilities were not investigated in the current study, and should be examined in future studies. The statistical robustness of the results in our study and previous studies should be considered carefully. Future studies investigating associations between ToM and negative symptoms at the behavioral and neural levels are warranted.

Our findings, linking deficits in inferring others' social emotions with lower activity in the left IFGs of patients with schizophrenia, are in line with the results of previous fMRI studies in patients with schizophrenia, using task demanding ability to infer others' emotions (de Achaval et al., 2012; Derntl et al., 2012; Russell et al., 2000; Smith et al., 2015). Although Lee et al. did 
not demonstrate significant differences in brain activity in the IFG when testing emotional empathy between schizophrenia and HC groups, they did show that the schizophrenia group had increased activity in the right middle/inferior frontal cortex under an inhibitory empathy condition from that in the HC group (Lee et al., 2010). An explanation for the negative finding in the IFG during emotional empathy is that unlike our task, which required the ability to infer social emotion, the task used by Lee et al. required the recognition of a basic emotion, like happiness, sadness, fear, or anger. The inhibitory empathy condition in their study therefore required a variety of cognitive components, including the ability to suppress the perspective of a person and provide a response that is incongruent with his/her intentions. Thus, their result is not directly comparable to ours. The current study showed no significant difference in activity in the right IFG between the HC and schizophrenia groups. This left IFG finding, with a significant difference in brain activity lacking in the right IFG, is concordant with findings of previous neuroimaging studies that reported functional abnormality in the left rather than in the right IFG among patients with schizophrenia (Kubicki et al., 2011).

During the process of inferring others' social emotions, the UHR group showed significantly lower bilateral IFG activity from that in the HC group. Although few fMRI studies have looked at the effects of tasks for inferring others' emotions among individuals at UHR, some neuroimaging studies have shown abnormalities in the IFG of these schizophrenia spectrum patients (Morey et al., 2005; Suzuki et al., 2005). Indeed, in a recent meta-analysis of multimodal neuroimaging studies for patients at genetic risk for developing schizophrenia, structural and functional abnormality was demonstrated in the IFG (Cooper et al., 2014). Further, we have also repeatedly reported a relationship between structural and functional change in this brain region and the clinical stage of psychosis (Iwashiro et al., 2012; Natsubori et al., 2014a; Suga et al., 2010; Yamasue et al., 2004). 
Several methodological limitations of the current study were of note. First, although psychotropic medications can modulate brain function, there was a difference in the daily antipsychotic dose between the UHR and schizophrenia groups. Since the current limited sample size did not allow us to test in the absence of antipsychotic medications, future studies should address the issue in neuroleptic-naïve patients. Second, we could not conduct this study with only male participants, even though sex differences have been suggested in social cognition and its neural underpinning (Yamasue et al., 2008). Third, although head motion during scanning is known to generate spurious findings, we have not excluded any participants due to excessive head motion during the scan. This is because the impact of head motion on findings is believed to be smaller in task-based fMRI compared with task-free neuroimaging, such as resting state fMRI and diffusion tensor imaging (Castellanos and Aoki, 2016). Fourth, although some previous studies in patients with schizophrenia or UHR with similar sample sizes have adopted a similar threshold as in the present study (e.g., Choi et al., 2012; Holt et al., 2011; Natsubori et al., 2014; Cremers et al., 2016), this chosen threshold is not stringent enough when small volume corrections are applied.

In summary, the current study revealed hyper-STS activity and hypo-IFG activity in patients with schizophrenia when engaged in inferring others' beliefs and/or emotions. During tasks designed to engage patients in inferring the beliefs of others, patients with schizophrenia showed significant difference in brain activity in both the left STS and the IFG, while brain activity of individuals at UHR were between those of patients with schizophrenia and HCs. In contrast, during tasks designed to engage patients in inferring the social emotions of others, individuals at UHR showed significantly altered brain activity in both the left STS and the right IFG, while patients with schizophrenia showed no such differences. The activity in the left IFG differed from normal in both the schizophrenia and UHR groups when engaged in inferring social 
Takano et al.

emotion. These findings might reflect different aspects of the same pathophysiological process at different clinical phases of psychosis. 


\section{Acknowledgements}

The current study was partially supported by Grants-in-Aid for Scientific Research (KAKENHI22689034/23659558/26670535) from the Japan Society for the Promotion of Science

(to HY); the Center-of-Innovation Program from Japan Science and Technology Agency and the Strategic Research Program for Brain Sciences by the Ministry of Education, Culture, Sports, Science and Technology (to HY). This work is also supported by the Japan Society for the Promotion of Science (to YA). 


\section{Contributors}

HY, NY, YK and YT designed the fMRI task and study protocol. SK and KK designed and managed the recruitment and clinical evaluation of the clinical participants of the study. HY, YT, YA, TN, HI, and NI collected the MRI and clinical data. HY, YA and YT managed the literature searches and analyses and wrote the manuscript. WG, HS and HT managed the MRI protocol and data collection. HY, NY, YA and YT contributed to analysis and interpretation of data. All the authors contributed to and approved the final manuscript. 


\section{References}

Aoki, Y., Yahata, N., Watanabe, T., Takano, Y., Kawakubo, Y., Kuwabara, H., Iwashiro, N., Natsubori, T., Inoue, H., Suga, M., Takao, H., Sasaki, H., Gonoi, W., Kunimatsu, A., Kasai, K., Yamasue, H., 2014. Oxytocin improves behavioural and neural deficits in inferring others' social emotions in autism. Brain 137, 3073-3086.

Baron-Cohen, S., Leslie, A.M., Frith, U., 1985. Does the autistic child have a "theory of mind"? Cognition 21, 37-46.

Benedetti, F., Bernasconi, A., Bosia, M., Cavallaro, R., Dallaspezia, S., Falini, A., Poletti, S., Radaelli, D., Riccaboni, R., Scotti, G., Smeraldi, E., 2009. Functional and structural brain correlates of theory of mind and empathy deficits in schizophrenia. Schizophr. Res. 114, 154-160.

Bora, E., Pantelis, C., 2013. Theory of mind impairments in first-episode psychosis, individuals at ultra-high risk for psychosis and in first-degree relatives of schizophrenia: systematic review and meta-analysis. Schizophr. Res. 144, 31-36.

Brune, M., Ozgurdal, S., Ansorge, N., von Reventlow, H.G., Peters, S., Nicolas, V., Tegenthoff, M., Juckel, G., Lissek, S., 2011. An fMRI study of "theory of mind" in at-risk states of psychosis: comparison with manifest schizophrenia and healthy controls. Neuroimage $55,329-337$.

Castellanos, F.X., and Aoki, Y. 2016. Intrinsic functional connectivity in attentiondeficit/hyperactivity disorder: A science in development. Biol. Psychiatry Cogn. Neurosci. Neuroimaging 1, 253-261.

Choi, J.S., Park, J.Y., Jung, M.H., Jang, J.H., Kang, D.H., Jung, W.H., Han, J.Y., Choi, C.H., Hong, K.S., Kwon, J.S., 2012. Phase-specific brain change of spatial working memory processing in genetic and ultra-high risk groups of schizophrenia. Schizophr. Bull. 38, 
1189-1199.

Cooper, D., Barker, V., Radua, J., Fusar-Poli, P., Lawrie, S.M., 2014. Multimodal voxel-based meta-analysis of structural and functional magnetic resonance imaging studies in those at elevated genetic risk of developing schizophrenia. Psychiatry Res. 221, 69-77.

Cremers, H., Lee, R., Keedy, S., Phan, KL., Coccaro, E. 2016. Effects of escitalopram administration on face processing in intermittent explosive disorder: an fMRI study. Neuropsychopharmacology 41, 590-597

Das, P., Lagopoulos, J., Coulston, C.M., Henderson, A.F., Malhi, G.S., 2012. Mentalizing impairment in schizophrenia: a functional MRI study. Schizophr. Res. 134, 158-164. de Achaval, D., Villarreal, M.F., Costanzo, E.Y., Douer, J., Castro, M.N., Mora, M.C., Nemeroff, C.B., Chu, E., Bar, K.J., Guinjoan, S.M., 2012. Decreased activity in right-hemisphere structures involved in social cognition in siblings discordant for schizophrenia. Schizophr. Res. 134, 171-179.

Derntl, B., Finkelmeyer, A., Voss, B., Eickhoff, S.B., Kellermann, T., Schneider, F., Habel, U., 2012. Neural correlates of the core facets of empathy in schizophrenia. Schizophr. Res. $136,70-81$.

Dodell-Feder, D., Tully, L.M., Lincoln, S.H., Hooker, C.I., 2014. The neural basis of theory of mind and its relationship to social functioning and social anhedonia in individuals with schizophrenia. Neuroimage Clin 4, 154-163.

Fett, A.K., Viechtbauer, W., Dominguez, M.D., Penn, D.L., van Os, J., Krabbendam, L., 2011. The relationship between neurocognition and social cognition with functional outcomes in schizophrenia: a meta-analysis. Neurosci. Biobehav. Rev. 35, 573-588.

First MB, Spitzer RL, Gibbon M, JBW., W., 1997. Structured clinical interview for DSM-IV axis I disorders, non-patient ed. Biometrics Research Department, New York State 
Psychiatric Institute, New York.

Frith, C.D., 1992. The cognitive neuropsychology of schizophrenia. Lawrence Erlbaum Associates Publishers.

Frith, C.D., 2004. Schizophrenia and theory of mind. Psychol. Med. 34, 385-389.

Green, M.F., Bearden, C.E., Cannon, T.D., Fiske, A.P., Hellemann, G.S., Horan, W.P., Kee, K., Kern, R.S., Lee, J., Sergi, M.J., Subotnik, K.L., Sugar, C.A., Ventura, J., Yee, C.M., Nuechterlein, K.H., 2012. Social cognition in schizophrenia, Part 1: performance across phase of illness. Schizophr. Bull. 38, 854-864.

Green, M.F., Lee, J., Ochsner, K.N., 2013. Adapting social neuroscience measures for schizophrenia clinical trials, Part 1: ferrying paradigms across perilous waters. Schizophr. Bull. 39, 1192-1200.

Green, M.F., Penn, D.L., 2013. Going from social neuroscience to schizophrenia clinical trials. Schizophr. Bull. 39, 1189-1191.

Harvey, P.O., Zaki, J., Lee, J., Ochsner, K., Green, M.F., 2013. Neural substrates of empathic accuracy in people with schizophrenia. Schizophr. Bull. 39, 617-628.

Hollingshead, A.B., 1965. Two-factor index of social position. New Haven: Yale University Press.

Holt, D.J., Cassidy, B.S., Andrews-Hanna, J.R., Lee, S.M., Coombs, G., Goff, D.C., Gabrieli, J.D., Moran, J.M., 2011. An anterior-to-posterior shift in midline cortical activity in schizophrenia during self-reflection. Biol. Psychiatry 69, 415-423.

Horan, W.P., Green, M.F., DeGroot, M., Fiske, A., Hellemann, G., Kee, K., Kern, R.S., Lee, J., Sergi, M.J., Subotnik, K.L., Sugar, C.A., Ventura, J., Nuechterlein, K.H., 2012. Social cognition in schizophrenia, Part 2: 12-month stability and prediction of functional outcome in first-episode patients. Schizophr. Bull. 38, 865-872. 
Iwashiro, N., Suga, M., Takano, Y., Inoue, H., Natsubori, T., Satomura, Y., Koike, S., Yahata, N., Murakami, M., Katsura, M., Gonoi, W., Sasaki, H., Takao, H., Abe, O., Kasai, K., Yamasue, H., 2012. Localized gray matter volume reductions in the pars triangularis of the inferior frontal gyrus in individuals at clinical high-risk for psychosis and first episode for schizophrenia. Schizophr. Res. 137, 124-131.

Kasai, K., Shenton, M.E., Salisbury, D.F., Hirayasu, Y., Onitsuka, T., Spencer, M.H., YurgelunTodd, D.A., Kikinis, R., Jolesz, F.A., McCarley, R.W., 2003. Progressive decrease of left Heschl gyrus and planum temporale gray matter volume in first-episode schizophrenia: a longitudinal magnetic resonance imaging study. Arch. Gen. Psychiatry $60,766-775$.

Kay, S.R., Fiszbein, A., Opler, L.A., 1987. The positive and negative syndrome scale (PANSS) for schizophrenia. Schizophr. Bull. 13, 261-276.

Kern, R.S., Penn, D.L., Lee, J., Horan, W.P., Reise, S.P., Ochsner, K.N., Marder, S.R., Green, M.F., 2013. Adapting social neuroscience measures for schizophrenia clinical trials, Part 2: trolling the depths of psychometric properties. Schizophr. Bull. 39, 1201-1210.

Kobayashi, H., Nozaki, S., Mizuno, M., 2007. Reliability of the structured interview for prodromal syndromes Japanese version (SIPS-J). Jpn. Bull. Soc. Psychiatry 15, 168174.

Koike, S., Takano, Y., Iwashiro, N., Satomura, Y., Suga, M., Nagai, T., Natsubori, T., Tada, M., Nishimura, Y., Yamasaki, S., Takizawa, R., Yahata, N., Araki, T., Yamasue, H., Kasai, K., 2013. A multimodal approach to investigate biomarkers for psychosis in a clinical setting: the integrative neuroimaging studies in schizophrenia targeting for early intervention and prevention (IN-STEP) project. Schizophr. Res. 143, 116-124.

Kubicki, M., Alvarado, J.L., Westin, C.F., Tate, D.F., Markant, D., Terry, D.P., Whitford, T.J., De 
Siebenthal, J., Bouix, S., McCarley, R.W., Kikinis, R., Shenton, M.E., 2011. Stochastic tractography study of Inferior Frontal Gyrus anatomical connectivity in schizophrenia. Neuroimage 55, 1657-1664.

Lee, S.J., Kang do, H., Kim, C.W., Gu, B.M., Park, J.Y., Choi, C.H., Shin, N.Y., Lee, J.M., Kwon, J.S., 2010. Multi-level comparison of empathy in schizophrenia: an fMRI study of a cartoon task. Psychiatry Res. 181, 121-129.

Lee, T.Y., Kim, S.N., Jang, J.H., Shim, G., Jung, W.H., Shin, N.Y., Kwon, J.S., 2013. Neural correlate of impulsivity in subjects at ultra-high risk for psychosis. Prog. Neuropsychopharmacol. Biol. Psychiatry 45, 165-169.

Matsuoka, K., Uno, M., Kasai, K., Koyama, K., Kim, Y., 2006. Estimation of premorbid IQ in individuals with Alzheimer's disease using Japanese ideographic script (Kanji) compound words: Japanese version of National Adult Reading Test. Psychiatry Clin. Neurosci. 60, 332-339.

Miller, T.J., McGlashan, T.H., Woods, S.W., Stein, K., Driesen, N., Corcoran, C.M., Hoffman, R., Davidson, L., 1999. Symptom assessment in schizophrenic prodromal states. Psychiatr. Q 70, 273-287.

Modinos, G., Renken, R., Shamay-Tsoory, S.G., Ormel, J., Aleman, A., 2010. Neurobiological correlates of theory of mind in psychosis proneness. Neuropsychologia 48, 3715-3724.

Morey, R.A., Inan, S., Mitchell, T.V., Perkins, D.O., Lieberman, J.A., Belger, A., 2005. Imaging frontostriatal function in ultra-high-risk, early, and chronic schizophrenia during executive processing. Arch. Gen. Psychiatry 62, 254-262.

Natsubori, T., Hashimoto, R., Yahata, N., Inoue, H., Takano, Y., Iwashiro, N., Koike, S., Gonoi, W., Sasaki, H., Takao, H., Abe, O., Kasai, K., Yamasue, H., 2014a. An fMRI study of visual lexical decision in patients with schizophrenia and clinical high-risk individuals. 
Schizophr. Res. 157, 218-224.

Natsubori, T., Inoue, H., Abe, O., Takano, Y., Iwashiro, N., Aoki, Y., Koike, S., Yahata, N., Katsura, M., Gonoi, W., Sasaki, H., Takao, H., Kasai, K., Yamasue, H., 2014 b. Reduced frontal glutamate + glutamine and $\mathrm{N}$-acetylaspartate levels in patients with chronic schizophrenia but not in those at clinical high risk for psychosis or with firstepisode schizophrenia. Schizophr. Bull. 40, 1128-1139.

Olbert, C.M., Penn, D.L., Kern, R.S., Lee, J., Horan, W.P., Reise, S.P., Ochsner, K.N., Marder, S.R., Green, M.F., 2013. Adapting social neuroscience measures for schizophrenia clinical trials, part 3: fathoming external validity. Schizophr. Bull. 39, 1211-1218.

Oldfield, R.C., 1971. The assessment and analysis of handedness: the Edinburgh inventory. Neuropsychologia 9, 97-113.

Pantelis, C., Yucel, M., Bora, E., Fornito, A., Testa, R., Brewer, W.J., Velakoulis, D., Wood, S.J., 2009. Neurobiological markers of illness onset in psychosis and schizophrenia: The search for a moving target. Neuropsychol. Rev. 19, 385-398.

Russell, T.A., Rubia, K., Bullmore, E.T., Soni, W., Suckling, J., Brammer, M.J., Simmons, A., Williams, S.C.R., Sharma, T., 2000. Exploring the social drain in schizophrenia: Left prefrontal underactivation during mental state attribution. Am. J. Psychiatry 157, 20402042.

Savla, G.N., Vella, L., Armstrong, C.C., Penn, D.L., Twamley, E.W., 2013. Deficits in domains of social cognition in schizophrenia: a meta-analysis of the empirical evidence. Schizophr. Bull. 39, 979-992.

Schmidt, S.J., Mueller, D.R., Roder, V., 2011. Social cognition as a mediator variable between neurocognition and functional outcome in schizophrenia: empirical review and new results by structural equation modeling. Schizophr. Bull. 37 Suppl 2, S41-54. 
Schurz, M., Radua, J., Aichhorn, M., Richlan, F., Perner, J., 2014. Fractionating theory of mind: a meta-analysis of functional brain imaging studies. Neurosci. Biobehav. Rev. 42, 9-34.

Senju, A., Southgate, V., White, S., Frith, U., 2009. Mindblind eyes: an absence of spontaneous theory of mind in Asperger syndrome. Science 325, 883-885.

Shamay-Tsoory, S.G., 2011. The neural bases for empathy. Neuroscientist 17, 18-24.

Shamay-Tsoory, S.G., Aharon-Peretz, J., Perry, D., 2009. Two systems for empathy: a double dissociation between emotional and cognitive empathy in inferior frontal gyrus versus ventromedial prefrontal lesions. Brain 132, 617-627.

Smith, M.J., Schroeder, M.P., Abram, S.V., Goldman, M.B., Parrish, T.B., Wang, X., Derntl, B., Habel, U., Decety, J., Reilly, J.L., Csernansky, J.G., Breiter, H.C., 2015. Alterations in brain activation during cognitive empathy are related to social functioning in schizophrenia. Schizophr. Bull. 41, 211-222.

Suga, M., Yamasue, H., Abe, O., Yamasaki, S., Yamada, H., Inoue, H., Takei, K., Aoki, S., Kasai, K., 2010. Reduced gray matter volume of Brodmann's Area 45 is associated with severe psychotic symptoms in patients with schizophrenia. Eur. Arch. Psychiatry Clin. Neurosci. 260, 465-473.

Sumich, A., Chitnis, X.A., Fannon, D.G., O'Ceallaigh, S., Doku, V.C., Faldrowicz, A., Sharma, T., 2005. Unreality symptoms and volumetric measures of Heschl's gyrus and planum temporal in first-episode psychosis. Biol. Psychiatry 57, 947-950.

Suzuki, M., Zhou, S.Y., Takahashi, T., Hagino, H., Kawasaki, Y., Niu, L., Matsui, M., Seto, H., Kurachi, M., 2005. Differential contributions of prefrontal and temporolimbic pathology to mechanisms of psychosis. Brain 128, 2109-2122.

Takahashi, T., Wood, S.J., Yung, A.R., Soulsby, B., McGorry, P.D., Suzuki, M., Kawasaki, Y., Phillips, L.J., Velakoulis, D., Pantelis, C., 2009. Progressive gray matter reduction of 
the superior temporal gyrus during transition to psychosis. Arch. Gen. Psychiatry 66, $366-376$.

Uetsuki, M., Matsuoka, K., Kim, Y., Araki, T., Suga, M., Yamasue, H., Maeda, K., Yamasaki, S., Furukawa, S., Iwanami, A., Kato, N., Kasai, K., 2006. Estimation of premorbid IQ by JART in schizophrenia. Seishin Igaku (Clin. Psychiatry) 48, 15-22.

Ventura, J., Ered, A., Gretchen-Doorly, D., Subotnik, K.L., Horan, W.P., Hellemann, G.S., Nuechterlein, K.H., 2015. Theory of mind in the early course of schizophrenia: stability, symptom and neurocognitive correlates, and relationship with functioning. Psychol Med 45, 2031-2043.

Ventura, J., Wood, R.C., Hellemann, G.S., 2013. Symptom domains and neurocognitive functioning can help differentiate social cognitive processes in schizophrenia: a metaanalysis. Schizophr Bull 39, 102-111.

Vollm, B.A., Taylor, A.N.W., Richardson, P., Corcoran, R., Stirling, J., McKie, S., Deakin, J.F.W., Elliott, R., 2006. Neuronal correlates of theory of mind and empathy: A functional magnetic resonance imaging study in a nonverbal task. Neuroimage 29, 90-98.

Yamasaki, S., Yamasue, H., Abe, O., Yamada, H., Iwanami, A., Hirayasu, Y., Nakamura, M., Furukawa, S., Rogers, M.A., Tanno, Y., Aoki, S., Kato, N., Kasai, K., 2007. Reduced planum temporale volume and delusional behaviour in patients with schizophrenia. Eur. Arch. Psychiatry Clin. Neurosci. 257, 318-324.

Yamasue, H., Abe, O., Suga, M., Yamada, H., Rogers, M.A., Aoki, S., Kato, N., Kasai, K., 2008. Sex-linked neuroanatomical basis of human altruistic cooperativeness. Cereb. Cortex $18,2331-2340$.

Yamasue, H., Iwanami, A., Hirayasu, Y., Yamada, H., Abe, O., Kuroki, N., Fukuda, R., Tsujii, K., Aoki, S., Ohtomo, K., Kato, N., Kasai, K., 2004. Localized volume reduction in 
prefrontal, temporolimbic, and paralimbic regions in schizophrenia: an MRI parcellation study. Psychiatry Res. 131, 195-207.

Zaki, J., Ochsner, K.N., 2012. The neuroscience of empathy: progress, pitfalls and promise. Nat. Neurosci. 15, 675-680. 


\section{Figure 1. fMRI results}

The left sub-panels show brain regions with the main effects by group (Healthy controls $[\mathrm{HC}] /$ Ultra-high-risk for psychosis [UHR]/Schizophrenia $[\mathrm{Sc}])$ in full factorial analysis and inferring others' beliefs and social emotions as contrasts (Voxel-threshold: uncorrected $P<0.001$, $\mathrm{k}>10$ ). The bar graphs on the right show the results from the subsequent post-hoc analyses in the left superior temporal sulcus (STS: [-50, -28, 2: Montreal Neurological Institute]) and inferior frontal gyrus (IFG: left $[-48,20,18]$; right $[46,28,22])(* P<0.05)$.

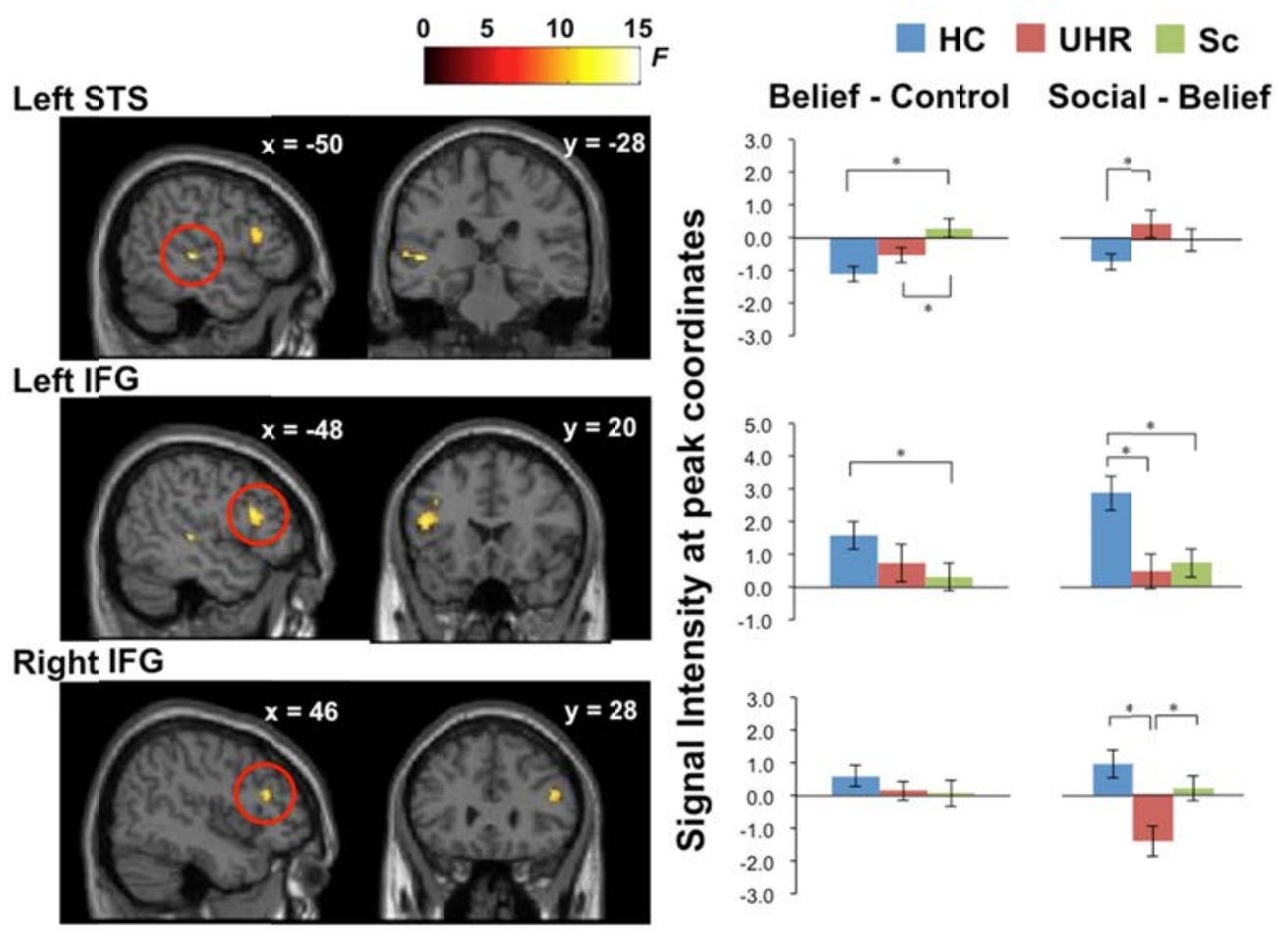


Table 1. Clinical and demographic characteristics of the study participants

\begin{tabular}{|c|c|c|c|c|c|c|c|c|}
\hline \multirow[b]{2}{*}{ Variable } & \multicolumn{2}{|c|}{$\begin{array}{l}\text { Individuals with } \\
\operatorname{UHR}^{\mathrm{a}}(\mathrm{n}=17)\end{array}$} & \multicolumn{2}{|c|}{$\begin{array}{c}\text { Patients with } \\
\text { schizophrenia } \\
\quad(\mathrm{n}=16)\end{array}$} & \multicolumn{2}{|c|}{$\begin{array}{l}\text { Healthy Controls } \\
\qquad(\mathrm{n}=20)\end{array}$} & \multicolumn{2}{|c|}{$\begin{array}{c}\mathrm{F} \text { Tests or } \mathrm{t} \text { Tests } \\
\text { or } \chi^{2}\end{array}$} \\
\hline & Mean & SD & Mean & SD & Mean & SD & $\begin{array}{l}\text { F or } \mathrm{t} \\
\text { value }\end{array}$ & $P$ \\
\hline Age & 22.4 & 4.1 & 25.5 & 6.5 & 24.7 & 4.3 & 1.7 & 0.19 \\
\hline Male / Female & $8 / 9$ & & $12 / 4$ & & $13 / 7$ & & $\chi^{2}=2.8$ & 0.24 \\
\hline Self socioeconomic status ${ }^{b}$ & 2.9 & 1.3 & 3.3 & 1.4 & 2.0 & 0.9 & 6.2 & $0.004 *$ \\
\hline Parental socioeconomic status ${ }^{\mathrm{b}}$ & 2.2 & 0.9 & 2.4 & 0.6 & 2.1 & 0.4 & 1.1 & 0.34 \\
\hline Estimated $\mathrm{IQ}^{\mathrm{c}}$ & 109.0 & 10.0 & 108.7 & 8.9 & 105.0 & 9.1 & 1.0 & 0.36 \\
\hline Handedness $^{\mathrm{d}}$ & 86.7 & 17.2 & 91.3 & 16.6 & 93.8 & 17.7 & 0.8 & 0.46 \\
\hline Antipsyochotic dose ${ }^{\mathrm{e}}$ (mg / day) & 157.2 & 218.0 & 408.0 & 376.2 & & & 2.4 & $0.025 *$ \\
\hline Age of Onset (year) & & & 23.1 & 6.1 & & & & \\
\hline Duration of untreated illness (weeks) & & & 21.8 & 27.9 & & & & \\
\hline Duration of illness (months) & & & 29.6 & 48.1 & & & & \\
\hline \multicolumn{9}{|l|}{ Positive and Negative Syndrome Scale } \\
\hline Positive symptoms & 13.3 & 3.6 & 14.3 & 4.6 & & & 0.7 & 0.48 \\
\hline Negative symptoms & 16.7 & 6.9 & 17.0 & 5.2 & & & 0.1 & 0.89 \\
\hline General psychology & 31.1 & 5.9 & 33.6 & 9.3 & & & 0.9 & 0.36 \\
\hline Delusional Behavior & 7.8 & 2.1 & 9.3 & 4.0 & & & 1.3 & 0.20 \\
\hline Global Assessment of Functioning & 48.9 & 11.6 & 46.6 & 16.9 & & & 0.5 & 0.65 \\
\hline
\end{tabular}

${ }^{\mathrm{a}}$ Ultra high-risk for schizophrenia; ${ }^{\mathrm{b}}$ Assessed using the Hollingshead scale. Higher scores indicate lower status; ${ }^{\mathrm{c}}$ Estimated from scores on the Japanese Adult Reading Test; ${ }^{\mathrm{d}}$ Assessed using the Edinburgh Inventory. $>0$ indicates right-handed; ${ }^{\mathrm{e}} \mathrm{Based}$ on chlorpromazine equivalents; ${ }^{*} P<0.05$ 
Table 2. Group differences in brain activity during understanding others' beliefs and social emotions

\begin{tabular}{|c|c|c|c|c|c|c|c|c|c|c|c|}
\hline \multirow{3}{*}{ Locations } & \multicolumn{5}{|c|}{ Main effects of group ${ }^{a}$} & \multicolumn{6}{|c|}{ Post-hoc analyses } \\
\hline & \multicolumn{3}{|c|}{ MNI coordinate } & \multirow{2}{*}{$\begin{array}{l}\text { Cluster } \\
\text { size** }\end{array}$} & \multirow{2}{*}{$Z$} & \multicolumn{3}{|c|}{$\begin{array}{c}\text { Social_emotion-minus- } \\
\text { Belief }\end{array}$} & \multicolumn{3}{|c|}{ Belief-minus-Control } \\
\hline & $\mathrm{x}$ & $\mathrm{y}$ & $\mathrm{z}$ & & & $\underset{\mathrm{n}}{\text { Compariso }}$ & $\mathrm{t}$ & $P$ & $\underset{\mathrm{n}}{\text { Compariso }}$ & $\mathrm{t}$ & $P$ \\
\hline \multirow[t]{2}{*}{ Right IFG } & 46 & 28 & 22 & 34 & 3.6 & $\mathrm{UHR}<\mathrm{HC}$ & 3.8 & 0.003 & & & \\
\hline & & & & & & $\mathrm{UHR}<\mathrm{Sc}$ & 2.7 & 0.011 & & & \\
\hline \multirow[t]{2}{*}{ Left IFG } & -48 & 20 & 18 & 130 & 3.8 & $\mathrm{UHR}<\mathrm{HC}$ & 3.2 & 0.003 & $\mathrm{Sc}<\mathrm{HC}$ & 2.1 & 0.043 \\
\hline & & & & & & $\mathrm{Sc}<\mathrm{HC}$ & 3 & 0.005 & & & \\
\hline \multirow[t]{2}{*}{ Left STG } & -50 & -28 & 2 & 54 & 4.1 & $\mathrm{HC}<\mathrm{UHR}$ & 2.6 & 0.014 & $\mathrm{HC}<\mathrm{Sc}$ & 3.8 & 0.001 \\
\hline & & & & & & & & & $\mathrm{UHR}<\mathrm{Sc}$ & 2.1 & 0.04 \\
\hline
\end{tabular}

IFG: Inferior frontal gyrus; STG: Superior temporal gyrus; MNI: Montreal Neurological Institute; UHR: Ultra-high risk; Sc: Schizophrenia; HC: Healthy control

${ }^{a}$ Main effects of contrast were shown in the Supplementary Table 2. No significant interaction between group and contrast was found.

**P $<0.001$, uncorrected 